in Rücksicht auf die in verschiedener Hinsicht vorhandene Gleichartigkeit der Eier für absolut einwandfrei halte. Das eine Gelege mit recht starken Eiern zeigt ausgesprochenen Hehertypus mit auffällig ammereierartigen Linieı und Schnörkeln, das andere Gelege mit rötlichem Untergrunde erinnert, abgesehen von seiner feinen Zeichnung, an die Eier unserer T. viscivorus oder an die japanischen Arten cardis und celaenops. Gröbere Fleckung mit heller erscheinenden Eiern innerhalb der Gelege kommt in mehr oder weniger ausgesprochener Deutlichkeit häufig vor, ausgesprochenen Hehertyp aber zeigen unter 17 Gelegen deren nur 2. Ein Gelege $z u \quad 4$ Stück enthält ein Riesenei mit folgenden Mafsen 33,0 × 24,2 mm, und das Ei eines anderen Geleges zeigt die Zeichnungsanhäufung am spitzen Pole. Dank der mir aus Gärten und Anlagen zahlreich zugegangenen verlassenen Gelege und das in der Dr. Proftschen Sammlung enthaltenen Materials kann ich den nachsteheuden Mafsen 86 Eier zu Grunde legen. Maximum 33, $2 \times 22,8 ; 31,7 \times 23,5 \mathrm{~mm}$, Minimum $25.4 \times 20,5$; $27,6 \times 20,0 \mathrm{~mm}$, Durchschnitt $29,8 \times 21,7 \mathrm{~mm}$.

\title{
Einige Beobachtungen und Verhörungen von der oberen Schara (Weifsrufsland.).
}

\section{Von Reinhold Fenk, Erfurt.}

Eine sich im Verhältnis zu der kurzen, nicht einmal zweijährigen Forschungszeit ganz stattlich ausnehmende "Liste der im Gebiete der Schara beobachteten Vögel" gab Graf Zedlitz im Reichenow - Ehrenband des Journ. f. Orn. 1917, des weiteren interessante biologische Beobachtungen dazu in seiner Abhandlung „Der Einflufs des russischen Winters auf die Vogelwelt" 1918 in selbiger Zeitschrift bringend. Ich war nun ein Jahr später als Zedlitz von Anfang April bis Weihnacht im gleichen Gebiet, mufste mich allerdings auf die grofsenteils sumpfigen, leider durch den Krieg gottsjämmerlich zerzausten Waldungen zwischen Ostrow und der Schara (Schtschara) sowie den Schützengraben oberhalb der sumfigen Wiese gleichen Flusses (beides unmittelbar beiderseitig der alten Napoleon'schen Heeresstrafse) beschränken, ohne selbst dieses, ich möchte sagen winzige Teilgebiet auch nur leidlich durchackern zu können, fehlte mir doch als einfachem M. G.-Schützen fast jedwede Bewegungsfreiheit. Schwerer fast noch wog, dafs ich aller ornithologischen Hilfsmittel, selbst eines Feldstechers bar war, sodafs ich mich in der Hauptsache auf Verhörungen beschränken mufste.

Gerade das letztere, worauf Graf Zedlitz wenig Wert legte, gibt mir aber Veranlassung, nachstehend einen Teil meiner Notizen auszugsweise zu bringen, soweit sie gewissermafen eine kleine 
Ergänzung, einen kleinen Nachtrag zu den Zedlitz'schen Abhandlungen bilden dürften, ansonst ich von der Veröffentlichung überhaupt abgesehen hätte.

Totanus ochropus L. P u nktierter oder W a ld wasserlä ufer.

Den Waldwasserläufer habe ich daheim so manches Mal im Frühling und Herbst, ja auch eine Zeit lang in Winters Mitten (vgl. Orn. Mtsber. 1912, p. 60) getroffen, mich stets ob seiner so klangvollen Flötenstrophen freuend. Jetzt so im rechteigentlichem Brutgelände der Art seiend, die nach Zedlitz in der ganzen Niederung nicht selten ist und die ich selbst an 4 Stellen traf (stets im Wald) - lernte ich mehr noch die Reichhaltigkeit und Modulationsfähigkeit seiner Rufe und Rufreihen, - lernte ich deren neue kennen, „schwungvoll accentuierte Tonreihen von silberhell flötendem Klange", wie Christoleit diese paarungszeitlichen Rufe einmal treffend bezeichnete. ${ }^{1}$ ) „Es sind - sagte Christoleit weiter - eigentlich drei Bestandteile, die in vielfacher Zusammensetzung und Modulation den Paarungsruf ausmachen: als Hauptton ein zweisilbiges hell flötendes „tlui“ oder tlüi“, das aber nie mehrmals unmittelbar aufeinanderfolgend wiederholt wird, der meist als Vorschlag zu diesem gebrauchte, seltener ihm, angehängte Lockton "titt" und endlich ein eigentlich nur eine Verlängerung des Locktones darstellendes „die“, das meist dem „tlui" angehängt wird und des Rythmus wegen auch zweisilbig am Ende absinkend (also "diëh") ausgesprochen werden kann."

In allem wollen sich meine Notizen diesem Schema nicht recht anpassen, die hauptsächlichen mögen hier folgen:

7./4. wütt deh ${ }^{\mathrm{i}}$ wütt, (dehi wütt, das dehi nach oben ge-

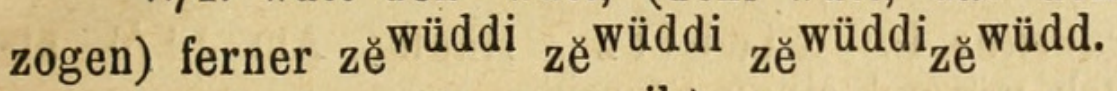

8./4. einer im Fluge tlu ${ }^{\text {iht }}$ pittpittpitt auf Kiefer aufbäumend; ab mit klick .... dann ein grillendes, hinaufgezogenes grüih und $\mathrm{tu}^{\mathrm{i}}$ dëwitt; zurückkommend wittwittwitt tudě ${ }^{\text {wih }}$ tudëwih tudéwih.

9./4. (an anderer Stelle) dreimaliges hinaufgezogenes tee ${ }^{\mathrm{i}-}$ (toi)wih , dann fortgesetztes widdiddi (wüddiddi mit Schlurstonwidd und klick (kick) ....

10/4. früh nach 4 Uhr schon fortgesetzte dè ${ }^{\text {widdi }}$ im Tannenmeisen-Rythmus, mittags wieder verschiedene Rufe $d 0^{i}$ wih; hittitti u. a.

15./5. wittwitt $\mathrm{tu}^{\mathrm{itt}} \mathrm{tu}^{\mathrm{itt}} \mathrm{tu}_{\text {tu }}$ (heraufgezogen, das tu wie immer betont).

1) Vgl. Orn. Mtschr. 1900, p. 485.

Journ, f, Orn, LXVLU, Jahrg. Juli/Oktober $1920^{\circ}$ 
4./6. f. tlu ${ }^{\mathrm{i}}$ titt.

Vom Ruf sagt Christoleit sehr richtig, dafs dieser paarungszeitlich nicht das dem Waldwasserläufer überall zugeschriebene (von den Zugästen bekannte - Fk.) dlüih-dlüidlüi, sondern ein einsilbiger kurzer, geschlossener heller Laut ,titt" sei. Auch ich notiere mir solchen meist so, aber auch mal als gipp ... oder klick und kick wie Dobbrick, auf wes letzteren in der Orn. Mtsschft. v. 1910 veröffentlichte Arbeit „Aus dem Leben des Waldwasserläufers" ich hinzuweisen nicht vergessen möchte.

1916 stellte Graf Zedlitz den ersten Heimwanderer am 10./4. fest, nachdem A. Marx die Art unmittelbar nach Beginn des Tauwetters am 20./3. bereits einmal traf. Als ich im folgenden Jahre am 7./4. einige Tage nach Beginn des Tauwetters nach einem neuen Sumpfwaldlager kam, fand ich mein dann täglich gehörtes Hauptpaar bereits vor, das ich im Übrigen stets nur flüchtig über den Wald segeln oder in den böchsten Regionen einer Kiefer aufbäumend verschwinden sah. Gebrütet hat es sicherlich, jedwede nähere diesbezl. Forschung hinderten indessen die Sümpfe.

Iringoides hypoleucos. Die bekannten Rufe hihd und hiddid diddi, auch mal tî̌llı, zuweilen als anschwellender und wieder sinkender hübscher Triller. Am 26./5. ein Paar mit 3-4 Jungen auf einer kleinen Schlammbank. Die Alte bildete, nachdem die Kleinen kückenartig untergeschlüpft waren (dabei leise hidd hidd fiebend), einen braunen, sich bis auf das Weifs in nichts von der Umgebung abhebenden, in der starken Dämmerung bald selbst für das platzkundige Auge ganz verschwindenden Ball.

Vanellus vanellus L. Kie bitz. Häufig. Am 14./6. attackierte ein Stück wütend einen Storch.

Gallinago gallinago L. B ek as s in e. Das meckernde twup- - hörte ich häufig und (auch zugleich damit) das uhrwerkmärsige, doch bald schneller, bald langsamer gebrachte ticke - - Am 19./6. bald nach Mitternacht rief ein Stück rick... tü ...

Crex pratensis. Den W a chtelk ön i g, der auf der Scharawiese fast den ganzen Tag über einzelner und nachts unaufhörlicher und vielfacher sein rähp rähp (am 13./5. rähprähp präh heruntersägte, lernte ich dank dieses Überfleifses ungemein schätzen, konnte ich mich doch so auf Posten ruhig meinen Träumereien - und die Wachsamkeit den Wiesenschnarrern überlassen, die ihr zwar nicht eben hübsches, dafür aber eigenartiges Konzert sicher eingestellt hätten, wenn die lieben Sibirier drüben sich zu einem unvermuteten Spaziergang über Flufs und Wiese entschlossen hätten. 
Cuculus canorus L. Den ersten Kuckuck stellte Graf Zedlitz 1916 für den 19./4. fest, im Übrigen schreibend: „Einen ähnlich dichten Besatz, gleichmäfsig über eine grofse Fläche verteilt, habe ich sonst nirgends gefunden." Ich vernahm 1917 den Erstling am 17. April, ab 21. dann mehrere hörend, und kann Zedlitz bestätigend nur sagen, dafs auch ich ein derartiges st attliches Kuckucksorchester noch nie sonst irgendwie hörte und - ich war doch gewifs schon in stark besiedelten Kuckucksgegenden. Ob jeweils $30,40,50$, ob mehr zugleich riefen, ich weils es nicht. Je zahlreicher eine Vogelart in einer Gegend, um so besser und vielgestaltiger gemeinhin der Gesang; - nun der klangvolle aber doch einfache Kuckucksruf ist gewifs nicht sonderlich variabel, aber was an Varianten in Bezug auf Höhe, Stimmung, Abtönung, hellerer oder dunklerer Klangschattierung und dergleichen aus ihm herauszuholen war, hörte ich sicher hier, und die Zusammenwirkung im Hall und Wiederhall! - Beschreiben läfst sich's nicht, ich kann nur sagen, dafs ich stundenlang meinen Posten überstand, den Morgenschlaf opferte, um dieses einzigartig wunderbare polyphone Kuckucksorchester zu hören. - Drei und viersilbige Rufe vernahm ich öfter, am 2./6. früh $3 \mathrm{Uhr}$ einen merkwürdigen Ruf und zwar folgte einen ,guggug“ stets eine Reihe von 4-6 und mehr kuckrŭ und $\operatorname{kuck}(e) r u ̆$, das ru unrein, kurz gestofsen.

Jynx torquilla, W e $\mathrm{nde} \mathrm{hals.} \mathrm{Ab} \mathrm{15./4.} \mathrm{fleifsig} \mathrm{rufend}$ (Zedlitz 1916 erstmalig 19./4., Grassmann in den etwas südlicher gelegenen Rokitnosümpfen am 18./4. ${ }^{1}$ ).

Dendrocopus major major L. Flügelm. eines am 14./12. frisch erl. Rotspecht-Q $144 \mathrm{~mm}$.

Cypselus apus apus L. Von Zedlitz nur als Durchzügler getroffen, da nirgends im Gebiete hobes Gemäuer. Ich vernahm die unverkennbaren schrillen Schreie am 21./6. sowie am 11. und 12./7. vormittags im Wald weit entfernt von irgend welchen Steingebäuden, erstfallsig 2 schnell über den Wald streichende Segler sehend.

Hirundo rustica rustica L. Immer wieder fielen mir die ausgesprochenen wie tui tui oder tuititt tuititt klingenden Stieglitztöne auf, die die Rauchschwalben der Schara vor dem ,witt witt" bezw, dem Zwitschern brachten. Die Klangfarbe glich so sehr den entsprechenden Tönen des Distelfinken, dafs ich tatsächlich die ersten zwei Male glaubte, letzteren vor einer Schwalbe zu hören, bis ich dann beides verbunden immer wieder von an und über mir vorbeischiefsenden Schwalben regelmäfsig vernahm .Noch am 26./9. traf ich übrigens Rauchschwalben.

1) Vgl. dessen Abh. „Zwei Jahre Feldorn, in den Rokitnosümpfen。. 
Ripària riparia riparia $\mathrm{L}$. U f e $\mathrm{s} \mathrm{ch}$ w a $1 \mathrm{~b}$ e. Im Flug durch die weifse Unterseite und das bekannte grä und schnelle schnerrende grägrägrägrä gut kenntlich.

Muscicapa grisola grisola L. Des g ra u en Fliegenfä n g e r s Gestammele hörte ich u. a. am 26. u. 27./5. aus Kiefern; an anderer Stelle, im sogenannten Sachsenwald notiere ich es mir am 3./6. mit ,iht iht ihtitt", auch wurde es dann länger ausgesponnen.

Muscicapa atricapilla atricapilla L. Zedlitz wie Marx konstatierten den Tra u ers c h n p p e r nur als Durchzügler. Ich fand ein Nest in ca. drei Meter Höhe in einer Pappel am 24./6. durch das fütternde Weibchen aufmerksam geworden. $\sigma^{\Upsilon}$ auch am Nest.

Muscicapa parva parva Bchst. Den Zwergfliegenfänger glaube ich einmal getroffen zu haben, dem Benehmen und flüchtigeren Sehen nach. Der Gesang erinnerte in etwas an Trauerschnepper, eine hübsche kleine Flötentour an Hänfling. Leider kam ich anderntags in Stellung, sodas positivere Beobachtungen wegfielen.

Lanius collurio collurio L. Zwei $\mathrm{r}$ o $\mathrm{tr}$ ü $\mathrm{c} \mathrm{k}$ i g e W ü $\mathrm{rg}$ e r, die ich an der Scharastellung regelmälsig traf, brachten beide neben dem bekannten rauhen "wä" fast stets ein merkwürdiges schnelles, dem Jungenbetteln ähnelndes „quitt . . . (w(u)itt, kitt o. ä.) und zwar sowohl vor oder nach dem arttypischen Ruf, als auch öfters als Gesangeseinleitung. - Übrigens waren es vorzügliche Spötter, die schier unübertrefflich im famosen langausgesponnenen Gesang u. a. ganze Flötenstropfen des Hänflings, Lerchenwirbeln, Schwalbengezwitscher, das "ninivé" und ,sisi brrr" der Kohlmeise, ganze Braunkehlchen- und Amselstrophen brachten. Das köstlichste bot sich mir indessen am 20./5. Ich lausche mit Genuls dem mir von der Heimat wohlbekannten hübschen, klaren Sang einer Sperbergrasmücke, als ich auch schon eine zweite aus meiner unmittelbaren Nachbarschaft höre und - zu meiner nicht geringen Ueberraschung das Echo sich als der Neuntöter entpuppt. Er bringt auf einer Buschspitze sitzend, wiederholend sein wä, wä, wä, dann wett ..., dem die Grasmückenstropfe in täuschendster Wiedergabe folgt. Gleich darauf singt auch die Sperbergrasmücke selbst wieder und diesmal erscheint mir ihr Lied etwas kräftiger, flötender, ob durch die Erregung? Vielleicht lernte der Würger von einer anderen oder er nahm das Lied in einiger Entfernung auf, es somit in der Wiedergabe etwas leiser bringend.

- Corvus corax corax L. Am 9./4 glaubte ich den Kolkraben getroffen $\mathrm{zu}$ haben. Am 15./4. vernehme ich ein merkwürdiges lautes Froschgequake „kŏ kŏ kŏ, dann kŏ qua“, dem ein ebenso merkwürdiges Gulgern folgte, das beides gehört zu haben, ich 
mich nicht entsinnen konnte, und doch, es wiederhörend, weifs ich mit grofser Deutlichkeit, das ich es irgendwo schon in einem Zoologischen Garten vernahm, sinne hin und her, ohne mich zunächst von den Amphibien oder anderen Sumpfbewohnern losreifsen zu können, bis ich mit einem Male wieder weifs, das es Kolkrabenrufe waren. Jetzt höre ich sie wieder ,korrrk .." im Weiterstreichen, von drüben eine bauchrednerische Antwort, dann rrrra, rrra, krrr $_{a}{ }_{a}$ und krrro $_{a}$ von ersterem, welches Krauchzen ich gleichfalls vom Zoo her kenne. - Auch am 24./4. höre ein Stück, es auch flüchtig, aber deutlich sehend.

Corvus cornix cornix L. Bin ich mit Kleinschmidt der Ansicht, dafs die Nebel- und Rabenkrähen in einen Formenkreis, eine Realgattung gehören, gab ich mir nichtsdestoweniger oder vielmehr gerade deswegen alle Mühe, irgend welche und seien es noch so geringfügige Unterschiede in den verschiedenen Rufen herauszuhören. All die verschiedenen Nebelkrähenrufe nun, die ich hörte, glichen indessen vollständig denen der Thüringer Rabenkrähen, mochte es das hölzerne tock ....., klingende $\operatorname{orrr}_{a}$, das helle $\operatorname{arrr}_{\mathrm{a}}$, krah und $\mathrm{k}^{\mathrm{u}}$ a, das tieere krahk o. a. sein. Man wird zugeben müssen, dafs das ein evidenter Beweis der Zusammengehörigkeit beider noch artlich getrennter Rassen ist, denn können schon artlich verschiedene, ja sogar recht verschiedene Tiere ein, zwei gleiche bezw. ähnliche Laute haben, so ist es doch klar, dafs wenn die Summe aller Töne, ja sogar der Varianten vollständig die gleiche ist, es sich oben nur um Geschöpfe e in e r S i p p $\theta$, um ein und dieselbe, nur äufserlich änderliche Art handeln mufs, zumal, wenn die Vögel geographisch weit genug getrennt sind, als dafs ein Nachspotten in Betracht kommen könnte, welches Nachspotten obendrein ja auch immer nur einen Teil, nie sämtliche Rufe erfafst hätte. gehört.

Oriolus oriolus o. L. Pirol. Erstmalig am 11./5.

Coccothraustes c. c. L. K e r n b e i $\int \mathrm{s}$ e r. 15./4. in Birken, zĕ zĕ iht iht (letzteres gequetscht hinaufgezogen). 12./5. kss kss (kiss) und iht.

Chloris c. c. L. Grün ling. 24./6. im Flug zit ... krihsch. 27. u. 31./5. Roller.

Carduelis c. c. L. Nach Zedlitz gemeiner Jahresvogel, den ich dagegen in meinem Gebiet trotz alles Aufmerkens auch nicht ein einziges Mal traf, allerdings war das Gelände auch weniger passend. Gesangesbruchstücke hörte ich übrigens spottend vom Braunkehlchen und im Übrigen täuschend ähnliche Stieglitzrufe immer wieder von Rauchschwalbe und Kuhstelze, wobei es sich natürlich nicht um Imitationen handelte. 
Chrysomitris spinus L. Der E rlz e i s i g, den Zedlitz nicht erwähnt, war dagegen für mein Gebiet Charaktervogel, den ich fast alltäglich im April, Mai, Juni und später an verschiedenen Stellen nicht nur des Nadelwaldes (Wettin- und Leipziger Lager, Sachsenwald u. a.), sondern auch am 22. u. 28./5. auf der Wiese an der Schtschara traf, jeweils durch die mir aus der Vogelstube u. der Thür. Heimat best bekannten Rufe, namentlich das charakteristische, etwas tannenmeisenartige tih, das jubelnde tǔliht sowie durch den Gesang mit der drolligen Krähstrophe (didel . . äätsch) aufmerksam geworden. Am 16./4. ein auffallend steinsperlingsoder grünfinkenartiges, nach oben im unreinen glissando hinaufgezogenes bäi, sodals ich im ersten Moment einen Grünfinken vermutete.

Acanthis linaria (subsp. ?) B i rk e $\mathrm{n} z$ e'i s i g. Im November und Dezember Trupps getroffen und das typische tscheck ... und hinaufgezogene main (ho ${ }^{\text {ihd) }}$ gehört.

Acanthis cannabina c. L. $\mathrm{H}$ ä $\mathrm{n} \mathrm{fl}$ ing. Häufig.

Pyrrhula p. p. Gim p el. Erst im Novbr. und Dezbr. und dann öfter gehört.

Loxia curvirostra c. L. Fichtenkreu $\mathrm{zschnabel}$. 25./4. gibb gibb gibibibipp; 5./5. gibibibibipp.

Fringilla coelebs $c$. L. B u c h f in k. Nicht genug konnte ich mich über das hundserbärmliche, zuweilen recht fitisähnliche Gestammele der Finken wundern, dafs ich über den halben April hinaus zu hören bekam, pflegen doch gerade die Finken grofser Nadelwälder gemeinhin die vorzüglichsten Schläger zu sein. Gegen und in der Hauptsache nach Mitte April hörte ich dann doch vortreffliche Schläger (öfter Würzgebühr); das matte, flackernde, fitisähnliche Stümpern der vorhergehende Zeit war, wie ich mir am 25./4. notierte, sicher nichts weiter als ein, unfertiges Dichten, Nichtdurchschlagen. Dieses merkwürdig späte Einsetzen des reifen, vollen Schlages wird erklärlich, wenn man Zedlitz Angabe liefst, dafs die Buchfinken anscheinend im Allgemeinen nicht in der Gegend überwintern, sondern sich erst mit Beginn des Tauwetters (1917 Anfang April) wieder einzustellen pflegen.

Passer domesticus $d$. L. Nicht wenig verwundert war ich über einige Haussperlinge, die ich in unserer Waldstellung traf. Wie in einigen hochgelegenen Thüringer Walddörfern der dort früher nicht ansässige Vogel sich einstellte, als dort die ersten Pferde gehalten wurden, dürften auch hier die Pferde bezw. deren Hinterlassenschaft der Anziehungspunkt gewesen sein.

Passer montanus $m$. L. Feldsperling. Bei einem kleinen Gütchen an einer grölserem, waldumgebenen Wiese. 
Emberiza calandra c. Gra u m m er. Nicht getroffen, da kein offenes Getreidegelände.

Emberiza citrinella erythrogenys Brehm." Zedlitz fand den Goldammer dem bäufigen sommerszeitlichen Vorkommen angemessen, als verhältnismäIsig spärlichen Überwinterer. Ich fand die Art, nach aus Anfang April stammenden Notizen recht häufig (das Tauwetter war inzwischen eingetreten) und ungemein variabel ihre Sangesweisen, was sicherlich seinen Grund zum Teil (ganz analog den Frühjarsverhältnissen bei uns) darin hatte, dafs es vielfach noch Strichgäste anderer Distrikte waren. Den heimischen Hauptschlag hörte ich damals nicht, bezw. in anderer Klangfarbe, am 20./5. (also wohl von einem sicheren Brutvogel) vernahm ich ihn dann erstmalig.

Ein sebr interessantes, im Pferdestall gefangenes $\sigma^{7}$ mit einem auffallend breiten, braunroten Wangenfleck und ohne Brustband bezw. nur solchem aus braunroten Federn (also mit Fichtenammerhinneigung) entwischte leider wieder.

Motacilla alba a. L. Das Reihen der Bachstelze hörte ich gleich bei meiner Ankunft wiederholt im Nadelwald, sah am 6./4. denn auch 2 Stück auf einem Kiefernstumpf, das typische ziwitt $\mathrm{u}$, psie, letzteres hier fast spatzenähnlich schilp klingend. $\mathrm{Am}$ 22./5. sah ich ein Nest mit 3 fast flüggen Jungen manneshoch in einem Horchpostengraben in einer kleinen, von oben etwas geschützten Lehmsandmulde. Alte beim Füttern meist auf die Zweige einer nahen Birke fliegend, von da auf den Grabenboden und dann hertrippelnd.

Motacilla flava (dombrowskii Tschusi?) Bei näher gesehenen Kuhstelzen fand ich wiederholt etwas fremdartiges am Kopf; dafs das nicht die heimische Form war, fühlte ich sofort, wufste aber wirklich nicht, wie ich ein Paar Stücke oder auch nur eines bekommen konnte. Daheim hätte ich nicht eher geruht, bis mir Klarheit geworden, hier waren mir ja wie in allem die Hände gebunden, und dieses in der Hauptsache so sinnlose Gefesseltsein von Geist, Willen und Können, der besten menschlichen Eigenschaften war es nicht zum Wenigsten, die mich bald in jene unheimliche Lustlosigkeit, Gleichgültigkeit und Schwermut verfallen liefsen.

Rufe der Kuhstelze ein rohrammerähnliches psi und psiě, aber etwas anders als daheim, auch das zilib hörte ich, indessen schärfer als bei uns; teilweise die Rufe an Kanarienvogel anklingend. Die oft direkt stieglitzartigen gestofsenen $p(u) i$ oder $\mathrm{pi}^{\mathrm{i}}$ erwähnte ich schon.

Anthus trivialis tr. L. Ab 17./4. (16./4?) gehört, die ersten Tage aber noch ohne Schlufsstrophe. 
Anthus pratensis L. Den Wiesenpieper glaubt Graf Zedlitz gesehen zu haben. Ich hörte zu Anfang April ein "niss niss" (Morast bei gröfserer Waldwiese) und 2 baumpieperähnliche Gesang esstrophen.

Lullula arborea subsp. Häufiger gehört.

Galerida cristata subsp. Am 20./5. und den folgenden Tagen hörte ich nur immer wieder den mir gänzlich unbekannten Ruf ti ${ }^{(i)}$ hinaufgezogen. Den hübschen typischen ganz andern heimischen Ruf entsinne mich, nie gehört zu haben.

Sitta europaea sordida Rchw. Unterschieden sich die gröfseren und unterseitig hellen Kleiber auffallend von der heimischen caesia, konnte ich stimmlich keinerlei Unterschiede finden.

Certhia familiaris familiaris $\mathrm{L}$. Die mich auch hier in der Klangfarbe sehr an Zaunkönig und Braunelle (allerdings feinklingiger), andrerseits an Blaumeise, kaum einmal entfernt an brachydactyla erinnernden Lieder des Nadelwaldbaumläufers glichen in allen Varianten denen der heimischen macrodactyla, ebenso natürlich die als ,z'ihss" und „ss ...." notierten Lockrufe. Dem so überaus charakteristischen tiddi tiriiddi; [Schema Hesse (Orn. Mtsber. 1907), Stadler und Schmitt (J. f. O. 1913) sisi sirisirisi (der letzte Ton ist oft wie bei Hesse angegeben niedriger als der vorletzte] gleichen diese zweithälftig oft perlenden, von mir im Scharagebiet u. a. mit sisisi sissie witt, ja sasse sissie sissississie witt, sisisi sissie sissie witt (der letzte pfeifende, öfter allerdings weggelassene Ton stets höher als der vorletzte) in Klangfarbe und namentlich Aufbau weit weniger als sich beispielsweise die Gesänge von Fitis und Waldlaubvogel, von Teich- und Sumpfrohrsänger gleichen und halte ich es für ausgeschlossen, dafs ein aufmerkender Mensch mit normalen Sinnen beide nicht zu unterscheiden vermag. Da, wo beide Arten nebeneinander vorkommen (im Allgemeinen wird sich familiaris bezw. macrodactyla an den Hochwald, namentlich Nadel- und Buchenwald, brachydactyla an die Parks und Gärten, den lichten Laubwald halten) und nur die eine bekannt oder bekannt gegeben ist, dürfte das stets daran liegen, dafs der betreffende Beobachter das ihm frühlenzzeitig alltägliche, markante Gartenbaumläuferliedchen wohl kennt, dafs des langkralligen Waldbaumläufers aber im vielstämmigen Wald überhört bezw. als Meisenliedchen verkennt, verkannt hat, am ehesten wohl als Blaumeisenliedchen, vielleicht auch als Finkenstümpern, Braunelle 0. a.

Certia brachydactyla hörte ich übrigens nicht im Scharagebiet, interessant ist, dafs Graf Zedlitz indessen soweit östlich den Vogel, der auch in Polen getroffen wurde, späterhin konstatierte.

Parus atricapillus borealis Selys $=\mathrm{Nord}$ is che Mat t $\mathrm{k}$ o $\mathrm{p} \mathrm{g} \mathrm{ra} \mathrm{u} \mathrm{m} \mathrm{i} \mathrm{s} \mathrm{e.} \mathrm{Traf} \mathrm{ich} \mathrm{Parus} \mathrm{atricapillus} \mathrm{salicarius,} \mathrm{die}$ 
heimische Mattkopf (Weiden-)Meise zwar im ganzen Thüringer Land wenn schon nichts weniger denn häufig, so doch immer wiederim Nadelwald sowie den Weidenreihen und -Wäldchen der Auen und Ströme, - hörte ich 1918 die wohlbekannten Rufe und Sangesweisen in all ihren Verschiedenheiten in den wildbuschigen, heckenumzäunten Obst- und Viehgärten bei Rozoy sur Serre (AisneDept. Frankreich) und in den belgischen Provinzen Limburg und Lüttich häufig und überall wieder, nur da von Parus a. subrhenanus, so erst recht in den grofsenteils (wenigstens zur Frühlingszeit) sumpfigen Misch- und Nadelwäldern des oberen Scharagebietes vom grofsen, hellen $\boldsymbol{P}$. $a$. borealis. Wenn nicht die Russen schliefslich doch noch subspecifisch von den Skandinaviern abzutrennen sind, was nach dem immerhin ganz reichlich verschiedenseitig vorliegenden Material nicht der Fall zu sein scheint, kommt diese Form in Betracht, da die Vögel der südlicher gelegenen Rokitnosümpfe nach den Stücken der Sammlungen Kleinschmidt und Görnitz zu ihr gehören, assimilis also nicht so weit heraufzugehen scheint und der allenfalls noch in Betracht kommen könnende $\boldsymbol{P}$. atricapillus bianchii (Sarudny und Härms) nach Kleinschmidts Untersuchungen an Härms'schen Vögeln nichts weiter als eine Aberration ist bezw. die Stücke durch Regen und Rindensaft in Baumlöchern verfärbte Exemplare von borealis sind. Wohl keinen Vogel hörte ich im ganzen oberen Scharagebiet so häufig und so regelmälsig fast die ganzeZeit hindurch als die Mattkopfmeise, und es war mir überaus iateressant, ganz die gleichen Rufe und Weisen wie daheim in all ihren Varianten und Modulationen (die selteneren hier gleicherweise selten) von einer, schon äufserlich selbst im Freien gut unterscheidbaren Form wieder zu vernehmen, so wie ich sie im Jahre danach von einer dritten Form (rhenanus bezw. subrhenanus), wie ich sie teilweise in flüchtiger Verhörung früher von einer vierten (submontanus in Bayr. Schwaben) zu hören bekam. Diese frappante Gleichheit der Lautäufserungen ist sicher eines der markantesten, nur leider noch kaum beachteten Kennzeichen der Artzusammengehörigkeit, auf das speciell zu achten es sich unsere Forscher angelegen sein lassen sollten. Hätten der verdienstvolle Herausgeber dieser Zeitschrift und Hesse so immer wieder durch die Bank die gleichen Lautäufserungen in und mit allen Varianten als ohrenfälligsten Beweis der Zusammengehörigkeit der Mattkopfmeisen Europa's zu hören bekommen, es wäre ihnen sicher nicht eingefallen in ihrer Neuen Namenliste der Vögel Deutschlands (J. f. O. 1916) eine Artrennung Salicarius mit subspecies rhenanus und Montanus mit borealis - damit scheinbar eine gleiche Trennung wie gegenüber Ater, Major, Palustris u. a. zu fixieren. Wie richtig es übrigens ist, die altweltlichen Mattkopfgraumeisen mit den amerikanischen in dem Formenkreis Atricapillus zu vereinigen, geht gleichfalls aus den Stimmenäufserungen bezw. den Angaben amerikanischer Forscher über die Rufe und Gesang von P. atri- 
capillus, carolinensis u. a. hervor. Es ist indessen hier nicht der Platz dies beweisend weiter auszuspinnen, möge dies einer späteren Arbeit in Kleinschmidt's Berajah-Salicarius-Monographie vorbehalten sein.

Einige Angaben über meine Verhörungen bei den ScharaMattköpfen mögen hier noch folgen. Ich sagte weiter oben schon, dafs die Lautäufserungen in ihrer Gesamtheit denen unserer heimischen Weidenmeisen glichen und so finde ich denn auch in meinen Notizen das rauhe einsilbige mit Zwischenpausen gebrachte , däh", das wesentlich hellere meist 4-6 fache ,deh .....", auch dehj und noch höher fast wie dih lautend, beides oft mit einem ein- bis vier- (meist zwei-) silbigem feinen si als Auftakt, letzteres auch öfters allein gebracht, ferner ein feines sitt sowie ein ganz blaumeisenartiges zittzitt als Rufäufserungen angegeben. Gleicherweise vernahm ich als Gesang die beiden mir von daheim bekannten Hauptformen und zwar meist das hübsche gestreckte, oft mit winzigen Vor- bezw. Nachschlägen bei jeder einzelnen Note (gegensätzlich zur Rufreihe, wo das sisi nur als Auftakt zur ganzen Reihe gebraucht wird) begleitete Pfeifen, einmal wie „zyje . . . ", ein anderes Mal "züzizizizih", dann wio dih . . ." oder mit den Vorschlägen „nz'dih nz'dih nz'dih“, „ě'tih ě'tih e’tih“ meist ,tih ...." klingend und dann auffallend dem melancholischen Gerufe des Waldschwirrers (Phyloscopus sibilator), welch' letzteres mir hier entschieden heller als das heimatliche „düh ......" erschien, ähnelnd, ebenfalls wie daheim dem (allerdings nur einsilbig gebracht werdenden) Lockruf des Erlzeisigs, dem Ausschlag des Baumpiepers und dem, mir freilich nur von Käfigvögeln her bekannten Pfeifen des Rötelammers (Emberiza rutila). War dies die Haupt- und, wennich mich recht entsinne, imSommersogar die einzige Weise, börte ich im Frühjahr fast häufiger das zuweilen an das Hämmern der Glanzkopfmeise ( $P$. palustris bezw. communis) sonst auch an die Mittelstrophe des Baumpiepers erinnernde Perlen als „dip .....", „zĭzĭž̆zǐzŭ zĕzězězĕ“ einmal zum Schlufs etwas fallend mit zip.... " notiert. Ferner hörte ich im zeitigen Frühjahr (also wie daheim auch) mal das eigenartige hübsche, etwas schnurrende, verschlungene "gürre".

Parus communis balticus Reichenow. G la n z köp fige $\mathrm{Grau}$ - alias N on enmeise. Schon länger hatte ich Bedenken gegen Beibehaltung des Namens ,palustris“, da sehr viel dafür spricht, das Linné damit den in Skandinavien häufigen "borealis" gemeint hat und es freut mich, dafs Kleinschmidt unabhängig davon zur gleichen Ansicht kam und Bestätigung fand: (vgl. Falco 1917, Ornis Germanica).

Die Nonnenmeise traf ich nur wenige Male. So am 17./7. beim Wettinlager durch das typische zji hähähähä aufmerksam geworden, ferner am 19./7. dann mal im Sept. sowie im Novbr. bei Podsokolje. 
Parus ater ater L. Graf Zedlitz sah die Tannenmeise nur vereinzelt während des Winters, und meint, dafs sie zur Brutzeit in den grofsen Nadelholzbeständen nicht so selten sein dürfte. Das sollte man auch wirklich mit aller Wahrscheinlichkeit annehmen, scheint aber überraschender Weise nicht der Fall zu sein, wenigstens habe auch ich den ja nach seiner charakteristischen Glöckchenstrophe so gut bestimmbaren Vogel auffallend wenig (nur Notizen vom 5. und 9./5.) getroffen, trotzdem ich mich gerade nach ihm umhörte, weil ich ihn vermifste. Sollte diese Spärlichkeit mit der Häufigkeit der mattköpfigen Graumeisen in irgend einem Zusammenhange stehen? Bei uns zu Lande ist ja das Verhältnis im Nadelwald das Umgekehrte.

Parus cristatus cr. L. $\mathrm{H}$ a u b e $\mathrm{n}$ e is e. Nächst borealis die häufigste Meise. Anfänglich wollte es mir scheinen, als sei das sisi girr weniger markant und schnurrend, das heifst fast ohne $r$. als das des heimischen $\boldsymbol{P}$. cristatus mitratus, notierte ich es u. a. doch mit sisi büi. Hörte ich nun im Anfang nur zufällig weicher rufende Stücke oder fiel mir der Unterschied später in der Alltäglichkeit nicht mehr auf?

Regulus regulus $r$. W in t e $\mathrm{g}$ ol d h ähn $\mathrm{ch}$ en. Kaum einige Male (so am 2./5. 6./5. und 5./6.) getroffen, obwohl solches doch eigentlich recht häufig im Gebiet sein mülste. Auch Zedlitz erwähnt nur eine brutzeitliche Beobachtung und bemerkt, dafs diese Art im Herbst und Winter ebenfalls nicht häufig sei.

Regulus ignicapillus $i$. T e m m. traf ich so wenig wie $\mathrm{Z}$.

Acrocephalus palustris B ech st. Von Graf Zedlitz nicht erwähnt, auch in den Rokitnosümpfen von Grafsmann nicht häufig beobachtet. Ich hörte nur am 9./6. bei Mondenschein, aber emfindlicher Kühle zwischen 1-2 Uhr nachts einige Stücke singen u. a. hauptsächliche Finkentöne, Gesangesbruchstück des Distelfinken und Rohrsängerartiges bringend nnd hörte selbabendlich gegen $7 \mathrm{Uhr}$ ein Stück in einem kleinen Buschwäldchen an anderer Stelle. Sicher handelte es sich um Durchzügler. Am 30./6. von Weitem gehört, vielleicht auch ein verspäteter Durchzügler oder Streifling.

Locustella naevia Bodd. H e u s c h r e c k e $\mathrm{n}$ s ä n g e r. Von Z. gleichfalls nicht erwähnt, in den Rokitnosümpfen dagegen nach Grafsmann weit verbreitet und recht zahlreich. Ich hörte am 12. und 13./5. flüchtig zweimal ein kurzes Bruchstück, beim Wettinlager (doch könnte es sich da um fluviatilis gehandelt haben) am 18./5. vollständig Sirren von Weitem, am 19./5. schwirrten auf den Schararwiesen zwischen 9-10 abends unaufhörlich mehrere in verschiedener Tonhöhe, meist in etwa 15 Sekunden langen Touren. Ich börte die Vögel dann öfter, so auch am 6./6. um die gleiche Zeit bei recht kühlem Winde. 
Rhylloscopus trochilus tr. L. Fitis. Ab 15./4. Weit bäufiger als bei uns perlten die Vögel ihre hübsche Pfeifstrophe nicht nur herab, sondern bogen nochmals auf und dann höher einsetzend also nochmals das herabperlen.

Phylloscopus collybita abietina Nils. Zilpzalp. 6./4. erstmalig, ab 9./4. häufig.

\section{Erlanger?) \\ Phylloscopus sibilator (erlangeri Hartert alias flavescens}

Als ein in der Tat ,sehr überraschendes und interessantes Ergebnis" mufs es bezeichnet werden, dafs Kleinschmidt ein am 15./5. 1916 von Dr. Schlüter im Kreise Smorgon gesammeltes Waldlaubvogelpärchen als zu dieser angeblicher und allgemeiner Annahme in den Mittelmeerländern und Südeuropa heimischen Form gehörig feststellte. Leider war es mir aus Eingangs geschilderten Gründen nicht möglich, Scharastücke zu bekommen, so häufig der Schwirrer gleichwohl und so fast handgreiflich vahe er mir oft war, im Übrigen nahm ich an, dafs die sonst in Rufsland sammelnden Ornithologen nun speciell auch ihr Augenmerk auf diese Vögel richten würden, um Füroder Gegenteiliges zur Klärung der Frage zu finden. Leider habe ich mich darin getäuscht! Mögen die einen Kleinschmidts Ausführungen übersehen oder nicht gekannt haben, betrachteten sie andere als absurd, anstatt einzudringen, sie mifsverstehend und es für ausgeschlossen betrachtend, dafs eine Form eine so merkwürdige Verbreitung zugleich in Nordafrika, Südeuropaund - dem nördlicheren Rufsland habenkönne. Dabei geht aus Kleinschmidts Ausführungen klipp und klar hervor, dafs er diese Waldlaubvögel (von denen er ein Stück vom 17./5. von Nordalgerien besitzt) als erst spät von Algerien (nicht wahrscheinlich im Mai durch Deutschland) nach Nordrufsland ziehende $G$ äste, nicht als $m e-$ diterrane Brutvögel betrachtet und schreibt: „Hartert suchte bekanntlich vergebens in Nordafrika nach Eiern".1) Von mir aus möchte ich dazu noch bemerken, dafs Hartert (Aus d. Wanderj. e. Naturf. u. Vögel pal. Fauna) angibt, der Vogel bringe nach seinen von Meade-Waldow und Witherby bestätigte Beobachtungen wohl ,eine flötende, aus den Locktönen ähnlichen Lauten zusammengesetzte Strophe (die ja allen Ornithologen bekannte dem Waldmeisenpfeifen ähnelnde Rufreihe düdüdüdüdüdü“ Fk.) nicht aber das Schwirren. Es wäre zu ergründen, ob diese Eigenümlichkeit konstant ist. Nun, wenn die Mittelmeerschwirrer tatsächlich das für Phylloscopus sibilator so unngemein typische

1) In s. Vög. pal. F. gibt Hartert an, dafs das Bruten in Nordafrika noch nicht mit Sicherheit festgestellt wurde, weiterhin, dafs Nester und Eier aus Südeuropa völlig denen der nördlicheren (mittel/westeur.) Form glichen. - Besitzt man dazugehørige Stucke und sind es tatsăchlich typische erlangeri? Fk. 
Schwirren nicht (oder vielleicht selten einmal) bringen, so wäre das nach meiner Ansicht ein gar nicht zu unterschätzender Beweis dafür, dafs die Vögel eben da nur weit von Heimat, Brunst und Brut entfernte Gäste sind. Wenigstens halte ich es nach allem ganz und gar ausgeschlossen, dafs eine Waldsängerform diesen markanten, so gern im arttypischen Schwobeflug gebrachten, die Art allerbestens kennzeichenden Gesang in der eigentlichen Brutheimat nicht bringen sollte.

Vielleicht wird man noch einwenden, dafs es sehr wenig plausibel erscheine solche bis mittmaiig in Nordafrika gefundene Stücke als nordische Gäste anzusehen, zumal wo bei uns der Waldschwirrer um mehr als Wochen-, ja Monatsfrist früher zurückzukehren pflegt. Meines Wissens erscheinen die Erstankömmlinge im südlichen Deutschland durchschnittlich vor und zu Anfang des zweiten Aprildrittels, in Mitteldeutschland nach Mitte, bis Ausgangs gleichen Monats, in den nördlicheren Teilen unseres Vaterlandes in den letzten April- bezw. den ersten Maitagen, übrigens mit wohl jeweils etwa vierzehntägigen Schwankungen in den verschiedenen Jahren. Zwischen Süd und Nord bezw. Nordost also schon bei uns eine Zeitspanne von mehreren Wochen! Sodann wolle man nicht vergessen, dafs zwischen diesen Erstankömmlingen und dem Häufigwerden der Art stets fast mehrere Tage, kommt eine Kältewelle mit Störung der Vegetationsentwickelung hinzu, sogar Wochen liegen; Wochen auch zwischen den Erstverhörungen und ersten Brutbeobachtungen. Einmal aufmerksam geworden, wird man ferner 3 ja 4 Wochen nach den Erstankömmlingen Waldlaubvögel vorübergehend in Gärten, Alleen, buschigen Berghängen u. dgl. - also nicht im typischen Brutgelände und darum auffallend - als Gäste bezw. Durchzügler bemerken, als Beispiel diene ein solcher flüchtiger Gartendurchzügler v. 3./5. 1918 b. Oreye (Prov. Lüttich) und ein heuer am 7./5. in Dederstedt in Fitisgesellschaft ${ }^{1}$ ) verhört und gesehenes Stück, während ich bei Erfurt die ersten dieses Jahres am 16./4., mehrere ab 19./4. konstatierte.

Mit eben diesen Erstbeobachtungen steht nun noch eine im scheinbaren Widerspruch, nämlich: Fand ich den Schwirrer seit dieser Zeit wohl im unterholzbelaubtem Steiger bei Erfurt,

1) Nach Kleinschmidt dort nicht brütend, aber im Mai in Fitisgesellschaft Zuggast. - Beim Fitis ist die Differenz gegentuber Erstbeobachtungen beim wenig mehr als $100 \mathrm{~km}$. südl. geleg. Erfurt v. 9. u. 10./4. noch auffallender. Noch interessanter ein gleichtagiges so verspatetes Wintergoldhahnchen. - Ein weiteres hochinteressantes Beispiel bilden 2 von mir am 16./5. im Erfurter Luisenpark angetroffene (schon am Benehmen als Fremdlinge erkennbare) Schwarzplättchen mit mehreren Sprosserbruchsttacken im Schlag, also sicher nordosst. Durchzügler aus Sprossergebieten. Beob. v. Sylvia atricapilla bei Erfurt ab 16./4, mithin ein Monat Unterschied! = 
so Anfang Mai nocht nicht im Buchenwald des nur wenige Kilometer entfernten Wildrodaer Forstes und nach Monatsfrist am 16./.5 (!) noch nicht in den allerdings wesentlich höher gelegenen etwa $40-50 \mathrm{Km}$. entfernten Buchenwaldungen zwischen Gehlberg und Oberhof, weil hier wie dort die Belaubung infolge der Kälteperiode auffällig zurückgeblieben war. Man sieht daraus zugleich, dafs das spätere Eintreffen des Vogels durch die Belaubung, die ja von Breitengrad zu Breitengrad nach Norden und Nordosten später zur Entwicklung kommt, bedingt ist, nicht etwa ein recht gemächliches Langsamwandern vorliegt.

Nach alledem erscheint es gewifs nicht mehr so absonderlich und unwahrscheinlich, dafs nordöstliche Waldschwirrer sich bis Mitte Mai noch in Nordafrika aufhalten können, zumal wenn man bedenkt, dafs so ein beschwingter Gesell, auch wenn er nicht gerade $\mathrm{zu}$ den Meisterfliegern seiner Zunft gehört und sich unterwegs allerhand Rast- und Futterpausen gönnt, doch immerhin nur Tagereisen bis zur Brutheimat benötigt. Einmal am Brutplatz angekommen, werden diese nördlicheren Vögel nach Analogieschlüssen wohl weniger Zeit vertrödeln, ehe sie zum Brüten schreiten als die Mitteleuropäer und selbst wenn, kann mithin ein Vogel, der beispielsweise wie der erwähnte Kleinschmidt'sche am 17./5. noch in Algier weilte, gut und gern in Nordrufsland zu Anfang Mitte Juni dem Nistgeschäft obliegen. Ohne die Helgoländer Durchzügler damit nun unbedingt verquicken zu wollen, möchte ich doch darauf hinweisen, dafs die Weigold'schen Beobachtungen von 1910, wo W. Waldschwirrer vom 13.-20. Mai (1909 nur ein Stück am 26./4, andersjähr. Beobachtungen fehlen leider) traf, recht schön dazu passen würden. Interessant übrigens die Weigold'sche Angabe, dafs 1910 infolge ,,andauernden Ostwindes" mehr Schwirrer über Helgoland gekommen seien. ${ }^{\mathbf{1}}$ )

Nur ein Bruchteil der $P h$. erlangeri bleibt wohl übrigens bis Mitte Mai in Nordafrika. Nach Hartert beobachtete man in Tunis, Algier undMarokko die, ,lebhaft gefärbte Form häufig im Frühling bis etwa Mitte Mai“. Während sie Hartert „am Oum R. Biah in Westmarokko anfangs April häufig fand, sandte Riggenbach von seinen nach dort im Mai unternommen Touren keine ein,

1) Welche unerwarteten grofsen Zeitdifferenzen im Durchzug einund derselben Vogelart liegen können, zeigen diese in vielem so interessanten Jahresberichte der Vogelwarte Helgoland in Masse. Schade, dafs man in Rositten all solchen doch gewils auch hoch interessanten Dingen, wie den Kleinvogelzug überhaupt, so wenig Interesse entgegen zu bringen scheint. Es wäre wirklich in so vielem wünschenswert, wenn Thienemann etwas entlastet würde und einen tüchtigen, jungen Assistenten bekäme, deren wir ja genug in Deutschland haben. Man hört ja immer wieder, dals die neue Regierung der Naturwissenschaft ganz anderes Interesse entgegenbringt und wăre die Schaffung solcher Assistentenstellen auf Anregung hin immerhin nicht ausgeschlossen. 
a uch Meade-Waldow erwähnt ihrer nicht aus dem Atlas, fand sie aber nach mündlichen Angaben in den Wäldern des mittleren Marrokko häufig (Vög. pal. F. p. 516.)

In der Ankunft der Osteuropäer, mindestens der der mittleren Breitegrade, dürften sich übrigens in fast allen die gleichen unterschiedlichen Zeitspannen geltend machen wie bei uns. Während Grafsmann 1916 den Waldlaubsänger in den Rokitnosümpfen (unt. 52. Breitengrad, $24 / 29^{0}$ östl. v. Gr.) bereits am $12 . / 4$. traf $^{1}$ ), Schelcher 1917 den ersten Gesang in Sarnki gorne (49 Br. 26 L..- $\left.{ }^{\circ}\right)$ am 10./4. vernahm, 2) hörte Zedlitz den Vogel 1916 im Scharagebiet $\left(53 / 26^{\circ}\right)$ erst von den letzten Apriltagen an, „dann allerdings ging ein balzen, jagen und raufen los". Ich hörte den Schwirrer $1916 \mathrm{im}$ Walde ob der oberen Schara ab 5. Mai, der Gesang war aber noch nicht fertig, noch ohne Schwirren, und zwar notierte ich mir ihn mit sisisisi sěsěsěsě, eine Phase mit absteigenden Tönen; am 6./5. vernahm ich hinwiederum im Fichtenwald mit vereinzelten Birken häufig die Sirrtöne allein, am 11./5. sibb ..... ih - . - -, dann sibb ..... sirrrr. Am 27./5. hörte ich den Vogel im reinen Kiefernwald ${ }^{3}$ ), ein ganzes Stück vom Mischwald entfernt und anscheinend nur als Gast (Nord. Durchzügler?). Das würde wieder sehr schön für das weiter oben Gesagte passen.

Vom ersten Tage an und immer wieder fiel mir auf, dafs das keinesfalls als Gesang aufzufassende bezw. mit dem Gesang zu verwechselnde, dem Weidenmeisenpfeifen oft sehr gleichende melancholische „tih - . - - - - - Gerufe tatsächlich deutlich dem Tannenmeisenruf ähnelnd „tih" zuweilen „tiě“" (oft eindringlich. scharf) klang und sich - entschieden heller und härter in der Klangfarbe - von dem bekannten weicheren heimischen „düh....“ auffallend genug unterschied. Da ich nun sicherlich nicht nur Scharabrutvögel sondern wohl auch nordische Durchzügler hörte, dürfte das konstant für die ost(nordost)europäischen Waldlaubsänger sein.

Am 11./5. vernahm ich von mehreren sich dicht vor mir im Baume herumtreibenden Waldlaubsängern schmatzende mir unbekannte Töne vor dem „tih".

Sylvia nisora. Bechst. Bezeichnet Grafsmann die Sperbergrasmücke als häufigste Grasmücke der Rokitnosümpfe, erwähnt

1) W. Grafsmann „der Frühjahrszug in den Rokitnosümpfen. O. Mschr. 1916 p. 233.

1919 p. 17.

2) R. Schelcher "Ornith. aus Galizien" Verh. d. Orn. Ges. in Bayern

$\left.{ }^{3}\right)$ Viele Angaben von des Voges Bräten im reinen Nadelwald dürften darauf basieren, dafs man ihn brutzeitlich darin antraf, singen börte und so als Brutrogel des betr. Geländes ansah, wăhrend or meist unr Streifling oder Durchzugagast gewesen sein dürfte. 
Zedlitz vom Scharagebiet nur ein, von einem überzähligen Männchen bis zu dessen Erlegung gestörtes Paar. Ich selbst kann die Art für mein Oberes Scharagebiet als nicht seltenen Vogel bezeichnen, der nicht nur an dem hängig-büschigen Teil des grofsen teils leider buschfreien Scharaufers siedelte (und zwar so alle 50-100 Meter ein Paar), sondern sich auch hinten in dem Moorwäldchen durch seinen hübschen Gesang und das charakteris tische Trommeln bemerkbar machte. Die Strophen waren ebenso hübsch und wechselreich wio daheim und ebenso schwer, ja in der Gesamtheit dank der Schnelligkeit gar nicht nachzuschreiben, wurden gleicherweise oft im Sitzen, Platzwechsel von Busch zu Busch bezw. arttypischen Singflug gebracht, nie im Hüpfen wie bei der Gartengrasmücke, deren Lied sie ja häufig zum Verwechseln ähneln, nur entschieden heller, weniger tieforgelnd und uicht so gebunden sind. Ein kürzeres Trommeln war des Öfteren eingeflochten, in Übrigen hörte ich dieses eigentümliche t(e)rrrrrr oder trrrrrah tettettettett häufig genug, weniger das Schnerren und würgerartige wä. Auch als Spötterin fand ich die Sperbergrasmücke, die anderseits derart trefflich vom rotrück. Würger imitiert wurde, dals ich oft zunächst nicht wufste, wer von beiden eigentlich der Sänger war.

Sylvia borin borin Bodd. Gar teng ras m ü cke. Von Zedlitz nicht erwähnt, im Rokitnogebiet von Grafsmann nur zweimal gesehen. Aus dem Schützengraben zurückkommend hörte ich die Art am 20./6. und später im Mischwald mit Unterholz unweit des Wettinlagers, sie schon an dem gurgelnd-plätschernden, gebundenen Gesang sofort von nisoria unterscheidend. Ersttägig nur kürzere, etwa nisorialange Strophen, dann aber auch das wundervolle lange einlullende Dahinplätschern.

Sylvia atricapilla und S. curruca. Schwarzplättchen (Mönchsgrasmücke) und Müllerchen scheint Graf Zedlitz gleicherweise nicht getroffen zu haben. Ich hörte beide erstmalig am 9./5. sie auch später hin und wieder an verschiedenen Stellen treffend.

Accentor (Prunella) modularis L. H ecke n b a u nelle. Jungfichtenbestände und anderes arttypisches Gelände in meinem Beobachtungsgebiet so gut wie nicht vorhanden, deshalb nur einmal im Fichtengebüsch zwischen Hochstammkiefern gehört.

Hypolais hypolais L. Gel bs p öt ter. Von Zedlitz nur einmal gehört und nach Grafsmann auch im Rokitnogebiet selten. Ich hörte in den ersten Junitagen ein Stück flüchtig, am 18./6. wiederholt eines beim Leipziger Lager (immer wieder die Reifstour u. a., aber nie das dedehoit), am 17. und 18./6. zwei Stück an verschiedenen Stellen unweit der Napoleon'schen Heeresstrafse.

Saxicola oenanthe oe. L. Das aufgewühlte Gelände hinter und zwischen den Gräben war ideal genug für den Steinschmätzer, als das er nicht häufig genug vorgekommen sein sollte, 
und so konnte ich mich auch öfter des Gaukelpfluges dieser hübschen Kerlchen erfreuen. Am 9./6. fing ich eins von zwei eben ausgeflogenen Pulli im Treppenloch eines Munitionsstollens, die wohl in dieser kaum einmal benutzten Erdstufe erbrütet worden waren.

Pratincola rubetra subsp. B ra un kehlchen. Verschiedene ausgezeichnete Spötter gehört.

Erithacus phoenicurus phoenicurus. Gartenrötling. Am 21./4. nach $4 \mathrm{Uhr}$ früh erstmalig gehört. Ein entzückend schön flötend-pfeifendes Stück, dessen Repertoire täglich reichhaltiger zu werden schien, erfreute mich dann wochenlang. Am 2./5. an anderer Stelle ein Stück getroffen.

Erithacus rubeculus $r$. L. Rotkehl chen. Am 8./4. erster Gesang von zwei Stück. Im Uebrigen zeigten sich die Rotkehlchen wie gemeinhin im grofsen Waldgebiet als wahre Meistersänger. Ein solch' langes im Anfang immer wieder in einigen Tönen steigendes und fallendes Lied von reinsten Pfeif- und Flötentönen, mit gestreckten Läufern, Trillern und Perlen habe ich wohl selten je so schön, wohl nie so lang gehört, als wie am 11./5. im Scharawald.

Erithacus (Luscinia) philomela Bechst. S p ros s e r. Aus einem kleinen trockenen Kiefernwäldchen, worin ich gewifs keinen Sprosser vermutet hätte, vernahm ich am 5./6. ein klangvolles zippenähnliches ,üht üht jüditt", dem ein an Nachtigall erinnerndes Knarren folgte. Immer wieder das drosselähnliche Jüdith, sonst noch ein Nachtigallperlen ,jick . . . . . . jack". Dieses abgerissene Stümpern eines späten Durchzüglers war das Einzige, was ich von dem König aller Sänger zu hören bekam, der nach den Erzählungen von Kameraden und nach Graf Zedlitz'Schilderung passenden Ortes recht häufig im Gebiet sein mufs, mindestens das zu seiner Zugzeit. Rechtschaffen hatte ich mich denn auch das ganze Frühjahr darauf gefreut, ihn ausgiebig in vielen Stücken verhören, richtig kennen lernen zu können, nun - was macht eine Enttäuschung mehr! - 


\section{$2 \mathrm{BHL}$ Biodiversity Heritage Library}

Fenk, Reinhold von. 1920. "Einige beobachtungen und verhörungen von der oberen schara (Weifsrufsland)." Journal $f u$

r Ornithologie 68, 308-325. https://doi.org/10.1007/bf02537889.

View This Item Online: $\underline{\text { https://www.biodiversitylibrary.org/item/43506 }}$

DOI: https://doi.org/10.1007/bf02537889

Permalink: https://www.biodiversitylibrary.org/partpdf/141106

\section{Holding Institution}

MBLWHOI Library

\section{Sponsored by}

MBLWHOI Library

\section{Copyright \& Reuse}

Copyright Status: No known copyright restrictions as determined by scanning institution.

This document was created from content at the Biodiversity Heritage Library, the world's largest open access digital library for biodiversity literature and archives. Visit BHL at https://www.biodiversitylibrary.org. 